\title{
$q$-Supercongruences from ${ }_{\|}$the $q$-Saalschütz identity
}

\author{
${ }^{1}$ Chuanan Wei, ${ }^{2}$ Yudong Liu, ${ }^{2}$ Xiaoxia Wang* \\ ${ }^{1}$ School of Biomedical Information and Engineering, \\ Hainan Medical University, Haikou 571199, China \\ ${ }^{2}$ Department of Mathematics, \\ Shanghai University, Shanghai 200444, China
}

\begin{abstract}
In terms of the $q$-Saalschütz identity and the Chinese remainder theorem for coprime polynomials, we establish some $q$-supercongruences modulo the third power of a cyclotomic polynomial. In particular, we give a $q$-analogue of a formula due to Long and Ramakrishna [Adv. Math. 290 (2016), 773-808].
\end{abstract}

Keywords: basic hypergeometric series; $q$-Saalschütz identity; $q$-supercongruence AMS Subject Classifications: 33D15; 11A07; 11B65

\section{Introduction}

For any complex variable $x$, define the shifted-factorial by

$$
(x)_{0}=1 \quad \text { and } \quad(x)_{n}=x(x+1) \cdots(x+n-1) \quad \text { when } n \in \mathbb{Z}^{+} .
$$

Let $p$ be an odd prime and $\mathbb{Z}_{p}$ denote the ring of all $p$-adic integers. Define Morita's $p$-adic Gamma function (cf. [14, Chapter 7]) to be

$$
\Gamma_{p}(0)=1 \quad \text { and } \quad \Gamma_{p}(n)=(-1)^{n} \prod_{\substack{1 \leqslant k<n \\ p \nmid k}} k, \quad \text { when } n \in \mathbb{Z}^{+} .
$$

Noting $\mathbb{N}$ is a dense subset of $\mathbb{Z}_{p}$ associated with the $p$-adic norm $|\cdot|_{p}$, for each $x \in \mathbb{Z}_{p}$, the definition of $p$-adic Gamma function can be extended as

$$
\Gamma_{p}(x)=\lim _{\substack{n \in \mathbb{N} \\|x-n|_{p} \rightarrow 0}} \Gamma_{p}(n) .
$$

Two important properties of the $p$-adic Gamma function can be expressed as follows:

$$
\frac{\Gamma_{p}(x+1)}{\Gamma_{p}(x)}= \begin{cases}-x, & \text { if } p \nmid x, \\ -1, & \text { if } p \mid x,\end{cases}
$$

The corresponding author*. Email addresses: weichuanan78@163.com (C. Wei), lydshdx@163.com (Y. Liu), xiaoxiawang@shu.edu.cn (X. Wang). 


$$
\Gamma_{p}(x) \Gamma_{p}(1-x)=(-1)^{\langle-x\rangle_{p}-1},
$$

where $\langle x\rangle_{p}$ indicates the least nonnegative residue of $x$ modulo $p$, i.e., $\langle x\rangle_{p} \equiv x(\bmod p)$ and $\langle x\rangle_{p} \in\{0,1, \ldots, p-1\}$. In 2006, Long and Ramakrishna [12, Proposition 25] proved that, for any prime $p$,

$$
\sum_{k=0}^{p-1} \frac{(1 / 3)_{k}^{3}}{k !^{3}} \equiv\left\{\begin{array}{lll}
\Gamma_{p}(1 / 3)^{6} & \left(\bmod p^{3}\right), & \text { if } p \equiv 1 \quad(\bmod 6) \\
-\frac{p^{2}}{3} \Gamma_{p}(1 / 3)^{6} & \left(\bmod p^{3}\right), & \text { if } p \equiv 5 \quad(\bmod 6)
\end{array}\right.
$$

For any complex numbers $x$ and $q$, define the $q$-shifted factorial by

$$
(x ; q)_{0}=1 \quad \text { and } \quad(x ; q)_{n}=(1-x)(1-x q) \cdots\left(1-x q^{n-1}\right) \quad \text { when } n \in \mathbb{Z}^{+} .
$$

For simplicity, we also adopt the compact notation

$$
\left(x_{1}, x_{2}, \ldots, x_{m} ; q\right)_{n}=\left(x_{1} ; q\right)_{n}\left(x_{2} ; q\right)_{n} \cdots\left(x_{m} ; q\right)_{n}
$$

Following Gasper and Rahman [1], define the basic hypergeometric series ${ }_{r+1} \phi_{r}$ to be

$$
{ }_{r+1} \phi_{r}\left[\begin{array}{c}
a_{1}, a_{2}, \ldots, a_{r+1} \\
b_{1}, b_{2}, \ldots, b_{r}
\end{array} ; q, z\right]=\sum_{k=0}^{\infty} \frac{\left(a_{1}, a_{2}, \ldots, a_{r+1} ; q\right)_{k}}{\left(q, b_{1}, b_{2}, \ldots, b_{r} ; q\right)_{k}} z^{k}
$$

Then the $q$-Saalschütz identity (cf. [1, Appendix (II.12)]) can be stated as

$$
{ }_{3} \phi_{2}\left[\begin{array}{c}
q^{-n}, a, b \\
c, q^{1-n} a b / c
\end{array} ; q, q\right]=\frac{(c / a, c / b ; q)_{n}}{(c, c / a b ; q)_{n}} .
$$

Recently, Guo [2, Theorem 1.1] found that, for positive integers $d, n, r$ such that $d \geq 2$, $n \equiv-r(\bmod d), n \geq d-r, r \leq d-2$, and $\operatorname{gcd}(d, r)=1$,

$$
\sum_{k=0}^{n-1} \frac{\left(q^{r} ; q^{d}\right)_{k}^{d}}{\left(q^{d} ; q^{d}\right)_{k}^{d}} q^{d k} \equiv 0 \quad\left(\bmod \Phi_{n}(q)^{2}\right)
$$

Here and throughout the paper, $\Phi_{n}(q)$ stands for the $n$-th cyclotomic polynomial in $q$ :

$$
\Phi_{n}(q)=\prod_{\substack{1 \leqslant k \leqslant n \\ \operatorname{gcd}(k, n)=1}}\left(q-\zeta^{k}\right)
$$

where $\zeta$ is an $n$-th primitive root of unity. For more $q$-analogues of supercongruences, we refer the reader to $[3-11,16,18,19]$.

Motivated by the work just mentioned, we shall establish the following two theorems. 
Theorem 1.1. Let $d \geq 2$ and $n$ be positive integers with $n \equiv 1(\bmod d)$. Then, modulo $\Phi_{n}(q)^{3}$

$$
\begin{aligned}
\sum_{k=0}^{(n-1) / d} \frac{\left(q ; q^{d}\right)_{k}^{3}}{\left(q^{3} ; q^{d}\right)_{k}\left(q^{d} ; q^{d}\right)_{k}^{2}} q^{d k} & \equiv q^{(n-1) / d} \frac{\left(q^{2}, q^{d-1} ; q^{d}\right)_{(n-1) / d}}{\left(q^{3}, q^{d} ; q^{d}\right)_{(n-1) / d}} \\
& \times\left\{1+[n]^{2} \sum_{i=1}^{(n-1) / d} \frac{q^{d i-d+2}}{[d i-d+2]^{2}}\right\},
\end{aligned}
$$

where $[n]=\left(1-q^{n}\right) /(1-q)$ is the q-integer.

Theorem 1.2. Let $n$ be a positive integer with $n \equiv 2(\bmod 3)$. Then, modulo $\Phi_{n}(q)^{3}$,

$$
\sum_{k=0}^{(2 n-1) / 3} \frac{\left(q ; q^{3}\right)_{k}^{3}}{\left(q^{3} ; q^{3}\right)_{k}^{3}} q^{3 k} \equiv q^{(2 n-1) / 3} \frac{\left(q^{2} ; q^{3}\right)_{(2 n-1) / 3}^{2}}{\left(q^{3} ; q^{3}\right)_{(2 n-1) / 3}^{2}}\left\{1-[2 n]^{2} \sum_{i=1}^{(2 n-1) / 3} \frac{q^{3 i-1}}{[3 i-1]^{2}}\right\} .
$$

Although we don't discover the general form of Theorem 1.2 including the parameter $d$, it is not difficult to understand that Theorem 1.1 with $d=3$ and Theorem 1.2 give a $q$-analogue of (1.1).

Letting $n=p$ be an odd prime and taking $q \rightarrow 1$ in the above two theorems, we obtain the following conclusion.

Corollary 1.3. Let $p$ be an odd prime such that $p \equiv t(\bmod 3)$ with $t \in\{1,2\}$. Then

$$
\sum_{k=0}^{(t p-1) / 3} \frac{(1 / 3)_{k}^{3}}{k !^{3}} \equiv \frac{(2 / 3)_{(t p-1) / 3}^{2}}{(1)_{(t p-1) / 3}^{2}}\left\{1+(-1)^{t-1}(t p)^{2} \sum_{i=1}^{(t p-1) / 3} \frac{1}{(3 i-1)^{2}}\right\} \quad\left(\bmod p^{3}\right)
$$

In order to explain the equivalence of (1.1) and (1.4), we need to verify the following relations.

Proposition 1.4. Let $p$ be an odd prime. Then

$$
\frac{(2 / 3)_{(p-1) / 3}^{2}}{(1)_{(p-1) / 3}^{2}}\left\{1+p^{2} \sum_{i=1}^{(p-1) / 3} \frac{1}{(3 i-1)^{2}}\right\} \equiv \Gamma_{p}(1 / 3)^{6} \quad\left(\bmod p^{3}\right)
$$

if $p \equiv 1(\bmod 3)$, and

$$
\frac{(2 / 3)_{(2 p-1) / 3}^{2}}{(1)_{(2 p-1) / 3}^{2}}\left\{1-4 p^{2} \sum_{i=1}^{(2 p-1) / 3} \frac{1}{(3 i-1)^{2}}\right\} \equiv-\frac{p^{2}}{3} \Gamma_{p}(1 / 3)^{6} \quad\left(\bmod p^{3}\right)
$$

if $p \equiv 2(\bmod 3)$.

The rest of the paper is arranged as follows. By means of (1.2) and the Chinese remainder theorem for coprime polynomials, a $q$-supercongruence modulo $\left(1-a q^{t n}\right)(a-$ $\left.q^{t n}\right)\left(b-q^{t n}\right)$ will be derived in Section 2. Then it is used to provide proofs of Theorems 1.1 and 1.2 in the same section. Finally, the proof of Proposition 1.4 will be displayed in Section 3. 


\section{Proofs of Theorems 1.1 and 1.2}

In order to prove Theorems 1.1 and 1.2 , we require the following united parameter extension of them.

Theorem 2.1. Let $d \geq 2, n$ be positive integers, $t \in\{1, d-1\}$ and $n \equiv t(\bmod d)$. Then, modulo $\left(1-a q^{t n}\right)\left(a-q^{t n}\right)\left(b-q^{t n}\right)$,

$$
\begin{aligned}
& \sum_{k=0}^{(t n-1) / d} \frac{\left(a q, q / a, q / b ; q^{d}\right)_{k}}{\left(q^{d}, c, q^{d+3} / b c ; q^{d}\right)_{k}} q^{d k} \\
& \quad \equiv \frac{\left(1-a q^{t n}\right)\left(a-q^{t n}\right)}{(a-b)(1-a b)} \frac{\left(c / a q, a c / q ; q^{d}\right)_{(t n-1) / d}}{\left(c, c / q^{2} ; q^{d}\right)_{(t n-1) / d}} \\
& \quad+\frac{\left(b-q^{t n}\right)\left(a b-1-a^{2}+a q^{t n}\right)}{(a-b)(1-a b)} \frac{(q / b)^{(t n-1) / d}\left(b c / q, q^{d+2} / c ; q^{d}\right)_{(t n-1) / d}}{\left(c, q^{d+3} / b c ; q^{d}\right)_{(t n-1) / d}}
\end{aligned}
$$

Proof. When $a=q^{-t n}$ or $a=q^{t n}$, the left-hand side of (2.1) is equal to

$$
\sum_{k=0}^{(t n-1) / d} \frac{\left(q^{1-t n}, q^{1+t n}, q / b ; q^{d}\right)_{k}}{\left(q^{d}, c, q^{d+3} / b c ; q^{d}\right)_{k}} q^{d k}={ }_{3} \phi_{2}\left[\begin{array}{c}
q^{1-t n}, q^{1+t n}, q / b \\
c, q^{d+3} / b c
\end{array} ; q^{d}, q^{d}\right] .
$$

Via (1.2), the right-hand side of (2.2) can be written as

$$
(q / b)^{(t n-1) / d} \frac{\left(b c / q, q^{d+2} / c ; q^{d}\right)_{(t n-1) / d}}{\left(c, q^{d+3} / b c ; q^{d}\right)_{(t n-1) / d}} .
$$

Since $\left(1-a q^{t n}\right)$ and $\left(a-q^{t n}\right)$ are relatively prime polynomials, we get the following result: modulo $\left(1-a q^{t n}\right)\left(a-q^{t n}\right)$,

$$
\sum_{k=0}^{(t n-1) / d} \frac{\left(a q, q / a, q / b ; q^{d}\right)_{k}}{\left(q^{d}, c, q^{d+3} / b c ; q^{d}\right)_{k}} q^{d k} \equiv(q / b)^{(t n-1) / d} \frac{\left(b c / q, q^{d+2} / c ; q^{d}\right)_{(t n-1) / d}}{\left(c, q^{d+3} / b c ; q^{d}\right)_{(t n-1) / d}}
$$

When $b=q^{t n}$, the left-hand side of (2.1) is equal to

$$
\sum_{k=0}^{(t n-1) / d} \frac{\left(a q, q / a, q^{1-t n} ; q^{d}\right)_{k}}{\left(q^{d}, c, q^{d+3-t n} / c ; q^{d}\right)_{k}} q^{d k}={ }_{3} \phi_{2}\left[\begin{array}{c}
a q, q / a, q^{1-t n} \\
c, q^{d+3-t n} / c
\end{array} ; q^{d}, q^{d}\right] .
$$

Through (1.2), the right-hand side of (2.4) can be evaluated as

$$
\frac{\left(c / a q, a c / q ; q^{d}\right)_{(t n-1) / d}}{\left(c, c / q^{2} ; q^{d}\right)_{(t n-1) / d}}
$$


Therefore, modulo $\left(b-q^{t n}\right)$,

$$
\sum_{k=0}^{(t n-1) / d} \frac{\left(a q, q / a, q / b ; q^{d}\right)_{k}}{\left(q^{d}, c, q^{d+3} / b c ; q^{d}\right)_{k}} q^{d k} \equiv \frac{\left(c / a q, a c / q ; q^{d}\right)_{(t n-1) / d}}{\left(c, c / q^{2} ; q^{d}\right)_{(t n-1) / d}} .
$$

It is clear that the polynomials $\left(1-a q^{t n}\right)\left(a-q^{t n}\right)$ and $\left(b-q^{t n}\right)$ are relatively prime. Noting the $q$-congruences

$$
\begin{aligned}
\frac{\left(b-q^{t n}\right)\left(a b-1-a^{2}+a q^{t n}\right)}{(a-b)(1-a b)} & \equiv 1 \quad\left(\bmod \left(1-a q^{t n}\right)\left(a-q^{t n}\right)\right), \\
\frac{\left(1-a q^{t n}\right)\left(a-q^{t n}\right)}{(a-b)(1-a b)} & \equiv 1 \quad\left(\bmod \left(b-q^{t n}\right)\right)
\end{aligned}
$$

and employing the Chinese remainder theorem for coprime polynomials, we arrive at Theorem 2.1 from (2.3) and (2.5).

Proof of Theorem 1.1. Letting $b \rightarrow 1, c \rightarrow q^{3}, t=1$ in Theorem 2.1, we obtain the formula: modulo $\Phi_{n}(q)\left(1-a q^{n}\right)\left(a-q^{n}\right)$,

$$
\begin{aligned}
& \sum_{k=0}^{(n-1) / d} \frac{\left(a q, q / a, q ; q^{d}\right)_{k}}{\left(q^{d}, q^{d}, q^{3} ; q^{d}\right)_{k}} q^{d k} \\
& \equiv q^{(n-1) / d} \frac{\left(q^{2}, q^{d-1} ; q^{d}\right)_{(n-1) / d}}{\left(q^{3}, q^{d} ; q^{d}\right)_{(n-1) / d}}+\frac{\left(1-a q^{n}\right)\left(a-q^{n}\right)}{(1-a)^{2}} \\
& \quad \times\left\{q^{(n-1) / d} \frac{\left(q^{2}, q^{d-1} ; q^{d}\right)_{(n-1) / d}}{\left(q^{3}, q^{d} ; q^{d}\right)_{(n-1) / d}}-\frac{\left(a q^{2}, q^{2} / a ; q^{d}\right)_{(n-1) / d}}{\left(q, q^{3} ; q^{d}\right)_{(n-1) / d}}\right\} .
\end{aligned}
$$

In term of the relation:

$$
q^{(n-1) / d} \frac{\left(q^{d-1} ; q^{d}\right)_{(n-1) / d}}{\left(q^{d} ; q^{d}\right)_{(n-1) / d}}=\frac{\left(q^{2-n} ; q^{d}\right)_{(n-1) / d}}{\left(q^{1-n} ; q^{d}\right)_{(n-1) / d}} \equiv \frac{\left(q^{2} ; q^{d}\right)_{(n-1) / d}}{\left(q ; q^{d}\right)_{(n-1) / d}} \quad\left(\bmod \Phi_{n}(q)\right),
$$

we get the conclusion: modulo $\Phi_{n}(q)\left(1-a q^{n}\right)\left(a-q^{n}\right)$,

$$
\begin{aligned}
& \sum_{k=0}^{(n-1) / d} \frac{\left(a q, q / a, q ; q^{d}\right)_{k}}{\left(q^{d}, q^{d}, q^{3} ; q^{d}\right)_{k}} q^{d k} \\
& \equiv q^{(n-1) / d} \frac{\left(q^{2}, q^{d-1} ; q^{d}\right)_{(n-1) / d}}{\left(q^{3}, q^{d} ; q^{d}\right)_{(n-1) / d}}+\frac{\left(1-a q^{n}\right)\left(a-q^{n}\right)}{(1-a)^{2}} \\
& \quad \times\left\{\frac{\left(q^{2}, q^{2} ; q^{d}\right)_{(n-1) / d}}{\left(q, q^{3} ; q^{d}\right)_{(n-1) / d}}-\frac{\left(a q^{2}, q^{2} / a ; q^{d}\right)_{(n-1) / d}}{\left(q, q^{3} ; q^{d}\right)_{(n-1) / d}}\right\} .
\end{aligned}
$$


By the L'Hôspital rule, we have

$$
\begin{aligned}
& \lim _{a \rightarrow 1} \frac{\left(1-a q^{n}\right)\left(a-q^{n}\right)}{(1-a)^{2}}\left\{\frac{\left(q^{2}, q^{2} ; q^{d}\right)_{(n-1) / d}}{\left(q, q^{3} ; q^{d}\right)_{(n-1) / d}}-\frac{\left(a q^{2}, q^{2} / a ; q^{d}\right)_{(n-1) / d}}{\left(q, q^{3} ; q^{d}\right)_{(n-1) / d}}\right\} \\
& \quad=[n]^{2} \frac{\left(q^{2} ; q^{d}\right)_{(n-1) / d}^{2}}{\left(q, q^{3} ; q^{d}\right)_{(n-1) / d}} \sum_{i=1}^{(n-1) / d} \frac{q^{d i-d+2}}{[d i-d+2]^{2}} .
\end{aligned}
$$

Letting $a \rightarrow 1$ in (2.7) and utilizing the above limit, we get the $q$-supercongruence: modulo $\Phi_{n}(q)^{3}$,

$$
\begin{aligned}
\sum_{k=0}^{(n-1) / d} \frac{\left(q ; q^{d}\right)_{k}^{3}}{\left(q^{3} ; q^{d}\right)_{k}\left(q^{d} ; q^{d}\right)_{k}^{2}} q^{d k} \equiv & q^{(n-1) / d} \frac{\left(q^{2}, q^{d-1} ; q^{d}\right)_{(n-1) / d}}{\left(q^{3}, q^{d} ; q^{d}\right)_{(n-1) / d}} \\
& +[n]^{2} \frac{\left(q^{2} ; q^{d}\right)_{(n-1) / d}^{2}}{\left(q, q^{3} ; q^{d}\right)_{(n-1) / d}} \sum_{i=1}^{(n-1) / d} \frac{q^{d i-d+2}}{[d i-d+2]^{2}}
\end{aligned}
$$

Substituting (2.6) into (2.8), we are led to Theorem 1.1 .

Proof of Theorem 1.2. Letting $b \rightarrow 1, c \rightarrow q^{3}, d=3, t=2$ in Theorem 2.1, we arrive at the result: modulo $\Phi_{n}(q)\left(1-a q^{2 n}\right)\left(a-q^{2 n}\right)$,

$$
\begin{aligned}
& \sum_{k=0}^{(2 n-1) / 3} \frac{\left(a q, q / a, q ; q^{3}\right)_{k}}{\left(q^{3} ; q^{3}\right)_{k}^{3}} q^{3 k} \\
& \equiv q^{(2 n-1) / 3} \frac{\left(q^{2} ; q^{3}\right)_{(2 n-1) / 3}^{2}}{\left(q^{3} ; q^{3}\right)_{(2 n-1) / 3}^{2}}+\frac{\left(1-a q^{2 n}\right)\left(a-q^{2 n}\right)}{(1-a)^{2}} \\
& \quad \times\left\{q^{(2 n-1) / 3} \frac{\left(q^{2} ; q^{3}\right)_{(2 n-1) / 3}^{2}}{\left(q^{3} ; q^{3}\right)_{(2 n-1) / 3}^{2}}-\frac{\left(a q^{2}, q^{2} / a ; q^{3}\right)_{(2 n-1) / 3}}{\left(q, q^{3} ; q^{3}\right)_{(2 n-1) / 3}}\right\} .
\end{aligned}
$$

Similar to the proof of (2.8), we can deduce from (2.9) that, modulo $\Phi_{n}(q)^{3}$,

$$
\begin{aligned}
\sum_{k=0}^{(2 n-1) / 3} \frac{\left(q ; q^{3}\right)_{k}^{3}}{\left(q^{3} ; q^{3}\right)_{k}^{3}} 3^{3 k} \equiv & q^{(2 n-1) / 3} \frac{\left(q^{2} ; q^{3}\right)_{(2 n-1) / 3}^{2}}{\left(q^{3} ; q^{3}\right)_{(2 n-1) / 3}^{2}} \\
& +[2 n]^{2} \frac{\left(q^{2} ; q^{3}\right)_{(2 n-1) / 3}^{2}}{\left(q, q^{3} ; q^{3}\right)_{(2 n-1) / 3}} \sum_{i=1}^{(2 n-1) / 3} \frac{q^{3 i-1}}{[3 i-1]^{2}}
\end{aligned}
$$

Moreover, it is routine to verify the congruence:

$$
\begin{aligned}
\left(q ; q^{3}\right)_{(2 n-1) / 3} & =(1-q)\left(1-q^{4}\right) \cdots\left(1-q^{2 n-3}\right) \\
& \equiv\left(1-q^{1-2 n}\right)\left(1-q^{4-2 n}\right) \cdots\left(1-q^{-3}\right) \\
& =(-1)^{(2 n-1) / 3} q^{-(n+1)(2 n-1) / 3}\left(q^{3} ; q^{3}\right)_{(2 n-1) / 3} \\
& \equiv-q^{-(2 n-1) / 3}\left(q^{3} ; q^{3}\right)_{(2 n-1) / 3} \quad\left(\bmod \Phi_{n}(q)\right) .
\end{aligned}
$$


The combination of (2.10) and (2.11) produces Theorem 1.2 .

\section{Proof of Proposition 1.4}

Now we begin to prove the supercongruence (1.5). Let $\Gamma_{p}^{\prime}(x)$ and $\Gamma_{p}^{\prime \prime}(x)$ respectively be the first derivative and second derivative of $\Gamma_{p}(x)$. By means of the properties of the $p$-adic Gamma function, we obtain

$$
\begin{aligned}
\frac{(2 / 3)_{(p-1) / 3}^{2}}{(1)_{(p-1) / 3}^{2}}= & \left\{\frac{\Gamma_{p}((1+p) / 3) \Gamma_{p}(1)}{\Gamma_{p}(2 / 3) \Gamma_{p}((2+p) / 3)}\right\}^{2} \\
= & \left\{\Gamma_{p}(1 / 3) \Gamma_{p}((1+p) / 3) \Gamma_{p}((1-p) / 3)\right\}^{2} \\
\equiv & \Gamma_{p}(1 / 3)^{2}\left\{\Gamma_{p}(1 / 3)+\Gamma_{p}^{\prime}(1 / 3) \frac{p}{3}+\Gamma_{p}^{\prime \prime}(1 / 3) \frac{p^{2}}{18}\right\}^{2} \\
& \times\left\{\Gamma_{p}(1 / 3)-\Gamma_{p}^{\prime}(1 / 3) \frac{p}{3}+\Gamma_{p}^{\prime \prime}(1 / 3) \frac{p^{2}}{18}\right\}^{2} \\
\equiv & \Gamma_{p}(1 / 3)^{6}\left\{1-\frac{2 p^{2}}{9} G_{1}(1 / 3)^{2}+\frac{2 p^{2}}{9} G_{2}(1 / 3)\right\} \quad\left(\bmod p^{3}\right)
\end{aligned}
$$

where $G_{1}(x)=\Gamma_{p}^{\prime}(x) / \Gamma_{p}(x)$ and $G_{2}(x)=\Gamma_{p}^{\prime \prime}(x) / \Gamma_{p}(x)$.

Let

$$
\begin{gathered}
H_{m}=\sum_{k=1}^{m} \frac{1}{k}, \quad H_{m}^{(2)}=\sum_{k=1}^{m} \frac{1}{k^{2}}, \\
H_{m}^{(2)}(p)=\sum_{\substack{1 \leq k \leq m \\
p \nmid k}} \frac{1}{k^{2}}, \quad \mathfrak{H}_{m}^{(2)}(p)=\sum_{\substack{1 \leq k_{1}<k_{2} \leq m \\
p \nmid k_{1} k_{2}}} \frac{1}{k_{1} k_{2}} .
\end{gathered}
$$

Via the following two relations from Pan, Tauraso and Wang [13, Theorem 4.1]:

$$
\begin{aligned}
G_{1}(1 / 3) & \equiv G_{1}(0)+H_{(2 p-2) / 3} \quad(\bmod p), \\
G_{2}(1 / 3) & \equiv G_{2}(0)+2 G_{1}(0) H_{(2 p-2) / 3}+2 \mathfrak{H}_{\left(2 p^{2}-2\right) / 3}^{(2)}(p) \quad\left(\bmod p^{2}\right)
\end{aligned}
$$

and the equation (cf. [17, Lemma 4.3]):

$$
G_{2}(0)=G_{1}(0)^{2}
$$

we get

$$
\begin{aligned}
G_{2}(1 / 3)-G_{1}(1 / 3)^{2} & \equiv 2 \mathfrak{H}_{\left(2 p^{2}-2\right) / 3}^{(2)}(p)-H_{(2 p-2) / 3}^{2} \\
& =-H_{\left(2 p^{2}-2\right) / 3}^{(2)}(p)
\end{aligned}
$$




$$
\begin{aligned}
& \equiv-\sum_{i=1}^{(2 p-2) / 3} \frac{1}{\left(i+\frac{2 p^{2}-2 p}{3}\right)^{2}} \\
& \equiv-H_{(2 p-2) / 3}^{(2)}(\bmod p) .
\end{aligned}
$$

In view of (3.1) and (3.2), the left-hand side of (1.5) is congruent to

$$
\begin{aligned}
& \Gamma_{p}(1 / 3)^{6}\left\{1-\frac{2 p^{2}}{9} H_{(2 p-2) / 3}^{(2)}\right\}\left\{1+p^{2} \sum_{i=1}^{(p-1) / 3} \frac{1}{(3 i-1)^{2}}\right\} \\
& \equiv \Gamma_{p}(1 / 3)^{6}\left\{1-\frac{2 p^{2}}{9} H_{(2 p-2) / 3}^{(2)}+p^{2} \sum_{i=1}^{(p-1) / 3} \frac{1}{(3 i-1)^{2}}\right\} \quad\left(\bmod p^{3}\right) .
\end{aligned}
$$

It is easy to see that

$$
\begin{aligned}
\sum_{i=1}^{(p-1) / 3} \frac{1}{(3 i-1)^{2}} & =H_{p-1}^{(2)}-\sum_{i=1}^{(p-1) / 3} \frac{1}{(3 i-2)^{2}}-\frac{1}{9} H_{(p-1) / 3}^{(2)} \\
& \equiv-\sum_{i=1}^{(p-1) / 3} \frac{1}{(3 i-2)^{2}}-\frac{1}{9} H_{(p-1) / 3}^{(2)} \\
& =-\sum_{i=1}^{(p-1) / 3} \frac{1}{(p-3 i)^{2}}-\frac{1}{9} H_{(p-1) / 3}^{(2)} \\
& \equiv-\frac{2}{9} H_{(p-1) / 3}^{(2)}=-\frac{2}{9} \sum_{i=(2 p+1) / 3}^{p-1} \frac{1}{(p-i)^{2}} \\
& \equiv-\frac{2}{9} \sum_{i=(2 p+1) / 3}^{p-1} \frac{1}{i^{2}} \equiv \frac{2}{9} H_{(2 p-2) / 3}^{(2)}(\bmod p)
\end{aligned}
$$

Substituting (3.4) into (3.3), we confirm the correctness of (1.5). The proof of (1.6) admits a similar process. The corresponding details are omitted here.

In 2015, Swisher [15, (H.3)] conjectured a nice supercongruence: for any prime $p \equiv 1$ $(\bmod 4)$,

$$
\sum_{k=0}^{\left(p^{r}-1\right) / 2} \frac{(1 / 2)_{k}^{3}}{k !^{3}} \equiv-\Gamma_{p}(1 / 4)^{4} \sum_{k=0}^{\left(p^{r-1}-1\right) / 2} \frac{(1 / 2)_{k}^{3}}{k !^{3}} \quad\left(\bmod p^{3 r}\right)
$$

where $r$ is a positive integer. On the basis of numerical calculations, we would like to put forward the following conjecture. 
Conjecture 3.1. Let $p$ be a prime with $p \equiv 1(\bmod 3)$ and $r$ a positive integer. Then

$$
\sum_{k=0}^{\left(p^{r}-1\right) / 3} \frac{(1 / 3)_{k}^{3}}{k !^{3}} \equiv \Gamma_{p}(1 / 3)^{6} \sum_{k=0}^{\left(p^{r-1}-1\right) / 3} \frac{(1 / 3)_{k}^{3}}{k !^{3}} \quad\left(\bmod p^{3 r}\right)
$$

\section{Acknowledgments}

The work is supported by the National Natural Science Foundations of China (Nos. 12071103 and 11661032).

\section{References}

[1] G. Gasper, M. Rahman, Basic Hypergeometric Series (2nd edition), Cambridge University Press, Cambridge, 2004.

[2] V.J.W. Guo, Factors of some truncated basic hypergeometric hypergeometric series, J. Math. Anal. Appl. 476 (2019), 851-859.

[3] V.J.W. Guo, $q$-Supercongruences modulo the fourth power of a cyclotomic polynomial via creative microscoping, Adv. Appl. Math. 120 (2020), Art. 102078.

[4] V.J.W. Guo, Proof of some $q$-supercongruences modulo the fourth power of a cyclotomic polynomial, Results Math. 75 (2020), Art. 77.

[5] V.J.W. Guo, A further $q$-analogue of Van Hamme's (H.2) supercongruence for primes $p \equiv 3$ (mod 4), Int. J. Number Theory, to appear.

[6] V.J.W. Guo and M.J. Schlosser, A new family of $q$-supercongruences modulo the fourth power of a cyclotomic polynomial, Results Math. 75 (2020), Art. 155.

[7] V.J.W. Guo, M.J. Schlosser, A family of $q$-hypergeometric congruences modulo the fourth power of a cyclotomic polynomial, Israel J. Math., to appear.

[8] V.J.W. Guo, M.J. Schlosser, Some $q$-supercongruences from transformation formulas for basic hypergeometric series, Constr. Approx., to appear.

[9] V.J.W. Guo, W. Zudilin, A q-microscope for supercongruences, Adv. Math. 346 (2019), 329-358.

[10] L. Li, S.-D. Wang, Proof of a $q$-supercongruence conjectured by Guo and Schlosser, Rev. R. Acad. Cienc. Exactas Fs. Nat., Ser. A Mat. RACSAM 114 (2020), Art. 190.

[11] J.-C. Liu, F. Petrov, Congruences on sums of $q$-binomial coefficients, Adv. Appl. Math. 116 (2020), Art. 102003.

[12] L. Long, R. Ramakrishna, Some supercongruences occurring in truncated hypergeometric series, Adv. Math. 290 (2016), 773-808.

[13] H. Pan, R. Tauraso, C. Wang, A local-global theorem for $p$-adic supergruences, preprint, 2020, arXiv:1909.08183v1.

[14] A.M. Robert, A Course in p-Adic Analysis, Graduate Texts in Mathematics, SpringerVerlag, New York, 2000. 
[15] H. Swisher, On the supercongruence conjectures of Van Hamme, Res. Math. Sci. 2 (2015), Art. 18.

[16] R. Tauraso, $q$-Analogs of some congruences involving Catalan numbers, Adv. Appl. Math. 48 (2009), 603-614.

[17] C. Wang, H. Pan, Supercongruences concerning truncated hypergeometric series, preprint, 2018, arXiv:1806.02735v2.

[18] X. Wang, M. Yue, Some $q$-supercongruences from Watson's ${ }_{8} \phi_{7}$ transformation formula, Results Math. 75 (2020), Art. 71.

[19] W. Zudilin, Congruences for q-binomial coefficients, Ann. Combin. 23 (2019), 1123-1135. 